УДК 517.55

V. V. QUAN, L. M. HAI

\title{
WEAK SOLUTIONS TO THE COMPLEX MONGE-AMPÈRE EQUATION ON OPEN SUBSETS OF $\mathbb{C}^{n}$
}

\author{
V. V. Quan, L. M. Hai. Weak solutions to the complex Monge-Ampère equation on open subsets \\ of $\mathbb{C}^{n}$, Mat. Stud. 51 (2019), 143-151. \\ In the paper, we prove the existence of weak solutions to the complex Monge-Ampère \\ equation in the class $\mathcal{D}(\Omega)$ on an open subset $\Omega$ of $\mathbb{C}^{n}$.
}

1. Introduction. As is well known, the complex Monge-Ampère operator plays a central role in pluripotential theory and has been extensively studied for many years. For a $\mathcal{C}^{2}$-smooth plurisubharmonic function $u$ defined on an open subset $\Omega$ of $\mathbb{C}^{n}$, its complex Monge-Ampère operator is defined by

$$
\left(d d^{c} u\right)^{n}=n ! 4^{n} \operatorname{det}\left(\frac{\partial^{2} u}{\partial z_{j} \partial \bar{z}_{k}}\right) d V_{2 n}
$$

where $d V_{2 n}$ is the volume form of $\mathbb{C}^{n}$. However, by an example in [21], Shiffman and Taylor have shown that it is impossible to extend the domain of definition of complex MongeAmpère operator in a meaningful way to the whole class of plurisubharmonic functions and still have the range contained in the class of non-negative Borel measures (see Appendix 1 in [21]). Bedford and Taylor [4] proved in 1982 that the complex Monge-Ampère operator is defined for locally bounded plurisubharmonic functions. Cegrell in [9] introduced and investigated in 2004 some classes of unbounded plurisubharmonic functions on bounded hyperconvex domains in $\mathbb{C}^{n}$. He has shown that the complex Monge-Ampère operator is well defined on the class $\mathcal{E}(\Omega)$ as a non-negative Radon measure and it is continuous on decreasing sequences of plurisubharmonic functions in this class. At the same time, in [9] he proved that the class $\mathcal{E}(\Omega)$ is the biggest class of plurisubharmonic functions with this property. To extend the class $\mathcal{E}(\Omega)$, in 2006, Błocki in [6] introduced the class $\mathcal{D}(\Omega)$ of plurisubharmonic functions on an open subset $\Omega$ of $\mathbb{C}^{n}$ and showed that the complex MongeAmpère operator can be well defined on this class. In the case $n=2$ he described this class by the equality: $\mathcal{D}(\Omega)=P S H(\Omega) \cap W_{\text {loc }}^{1,2}(\Omega)$ (see Theorem 1.1 in [5]), where $W_{\text {loc }}^{1,2}(\Omega)$ is the Sobolev space on $\Omega$. He also showed that if $\Omega$ is a bounded hyperconvex domain then $\mathcal{E}(\Omega)=P S H^{-}(\Omega) \cap \mathcal{D}(\Omega)$. Therefore, by results of Błocki in [6] one knows that the class $\mathcal{D}(\Omega)$ is the biggest class on which the complex Monge-Ampère operator is well-defined when $\Omega$ is an open subset of $\mathbb{C}^{n}$.

2010 Mathematics Subject Classification: 32U05, 32W20.

Keywords: Monge-Ampère equation; weak solution; plurisubharmonic function; hyperconvex domain. doi:10.15330/ms.51.2.143-151

(C) V. V. Quan, L. M. Hai, 2019 
One of the important and central problems of pluripotential theory is to define weak solutions to the complex Monge-Ampère equation. Namely we consider the following problem. Let $\mu$ be a non-negative Radon measure defined in an open subset $\Omega$ of $\mathbb{C}^{n}$. The problem is to find $w \in P S H(\Omega)$ such that

$$
\left\{\begin{array}{l}
w \in \mathcal{D}(\Omega), \\
\left(d d^{c} w\right)^{n}=\mu \text { in } \Omega .
\end{array}\right.
$$

When $\Omega$ is a strictly pseudoconvex domain in $\mathbb{C}^{n}$, there are some known results concerning to weak solutions of (1). Bedford and Taylor in [3] proved in 1976 that if $\mu=f d V$ with $f \in \mathcal{C}(\bar{\Omega})$ then $(1)$ has a continuous solution on $\bar{\Omega}$. Kołodziej in [20] showed in 1995 that if there exists a bounded subsolution for (1) then we can find $u \in P S H \cap L^{\infty}(\Omega)$ with $\left(d d^{c} u\right)^{n}=\mu$ (see Theorem $\mathrm{A}$ in [20]).

In the case $\Omega$ is a bounded hyperconvex domain in $\mathbb{C}^{n}$, the problem becomes much more complicated. Cegrell in [8] considered in 1998 weak solutions of the above problem for unbounded plurisubharmonic functions. In [9] he proved that if $\Omega$ is a bounded hyperconvex domain and $\mu$ vanishes on all pluripolar sets of $\Omega$ then (1) has a unique weak solution in the class $\mathcal{F}^{a}(\Omega)$ (see Theorem 5.14 in [9]). Next, Åhag, Cegrell, Czyż and Hiep in [1] studied in 2009 the above problem for non-negative measures carried by a pluripolar set. They claimed that if $\mu \leq\left(d d^{c} u\right)^{n}$ where $u \in \mathcal{E}(\Omega)$ then for every $H \in \mathcal{E}(\Omega) \cap M P S H(\Omega)$ there exists a weak solution $w \in \mathcal{E}(\Omega, H)$ such that $H+u \leq w \leq H$ satisfying (1), where $\operatorname{MPSH}(\Omega)$ denotes the set of maximal plurisubharmonic functions on $\Omega$.

Next, the problem (1) is also studied by some authors under different hypotheses for $\Omega$ and the measure $\mu$. In [12], the authors solved the above problem when $\Omega$ is a bounded domain and $\mu$ vanishes on pluripolar sets. When $\Omega$ is an unbounded pseudoconvex domain in $\mathbb{C}^{n}$, in [2] Åhag and Czyż treated the problem (1). They showed that Kołodziej's subsolution theorem is also valid. Next, in the case $\Omega$ is an unbounded hyperconvex domain, the problem (1) is studied by L.M.Hai, N.V.Trao and N.X.Hong. In [13] under the assymption that $\Omega$ is an unbounded hyperconvex domain and $\mu$ is a Borel measure on $\Omega$ with $\mu(\Omega)<\infty$ the authors proved that there exists a weak solution in the Cegrell class with boundary values $\mathcal{F}(\Omega, f)$ (see Theorem 5.3). The main aim of this paper is to establish the existence of weak solutions to the equation (1) in the case $\Omega$ is an arbitrary open subset of $\mathbb{C}^{n}$. Namely, we prove the following.

Theorem 1. Let $\Omega$ be an open subset of $\mathbb{C}^{n}$ and $u \in \mathcal{D}(\Omega), v \in M P S H(\Omega)$ be such that $u \leq v$ in $\Omega$. If $\mu \leq\left(d d^{c} u\right)^{n}$ then there exists a solution $w$ to (1) satisfying $u \leq w \leq v$ in $\Omega$.

It should be remarked that to find weak solutions of equation (1) for this case the methords that we use are different from that given by the authors in [12], [13], in particular, [2]. Here we combine results and techniques in the class $\mathcal{D}(\Omega)$ with results obtained in pluripotential theory on Cegrell's classes to claim the existence of desired weak solutions of the above equation on an arbitrary open subset $\Omega$ in $\mathbb{C}^{n}$.

The organization of the paper is as follows. In Section 2 we recall some notions of pluripotential theory which is necessary for the next results of the paper. In Section 3 we prove Theorem 1.

2. Preliminairies. In this section, we recall some elements of pluripotential theory that will be used throughout the paper. All this can be found in [1]-[20]. Let $n$ be a positive integer and 
let $\Omega$ be an open set in $\mathbb{C}^{n}$. We denote by $P S H(\Omega)$ the family of plurisubharmonic functions defined on $\Omega$ and $P S H^{-}(\Omega)$ denotes the set of negative plurisubharmonic functions on $\Omega$. We first recall the definition of the class $\mathcal{D}(\Omega)$ on an open set $\Omega$ in $\mathbb{C}^{n}$ (see [6]).

Definition 1. A plurisubharmonic function $u$ defined on $\Omega$ belongs to $\mathcal{D}(\Omega)$ if there exists a nonnegative Radon measure $\mu$ on $\Omega$ such that if $\Omega^{\prime} \Subset \Omega$ is an open subset and $\left\{u_{j}\right\} \subset$ $P S H\left(\Omega^{\prime}\right) \cap \mathcal{C}^{\infty}\left(\Omega^{\prime}\right)$ is a sequence which decreases to $u$ in $\Omega^{\prime}$ then $\left(d d^{c} u_{j}\right)^{n}$ tends weakly to $\mu$ in $\Omega^{\prime}$. The measure $\mu$ is denoted by $\left(d d^{c} u\right)^{n}$.

Note that by results of Bedford-Taylor in [4] we have $P S H(\Omega) \cap L_{\text {loc }}^{\infty}(\Omega) \subset \mathcal{D}(\Omega)$. Moreover, if $n=1$ then $S H(\Omega)=\mathcal{D}(\Omega)$.

Next, we recall the following classes of plurisubharmonic functions introduced and investigated by Cegrell in [9] in the case when $\Omega$ is a bounded hyperconvex domain in $\mathbb{C}^{n}$.

Definition 2. Let $\Omega$ be a bounded hyperconvex domain in $\mathbb{C}^{n}$. We say that a bounded, negative plurisubharmonic function $\varphi$ on $\Omega$ belongs to $\mathcal{E}_{0}(\Omega)$ if $\{\varphi<-\varepsilon\} \Subset \Omega$ for all $\varepsilon>0$ and $\int_{\Omega}\left(d d^{c} \varphi\right)^{n}<+\infty$.

Let $\mathcal{F}(\Omega)$ be the family of plurisubharmonic functions $\varphi$ defined on $\Omega$, such that there exists a decreasing sequence $\left\{\varphi_{j}\right\} \subset \mathcal{E}_{0}(\Omega)$ that converges pointwise to $\varphi$ on $\Omega$ as $j \rightarrow \infty$ and

$$
\sup _{j} \int_{\Omega}\left(d d^{c} \varphi_{j}\right)^{n}<\infty
$$

We denote by $\mathcal{E}(\Omega)$ the family of plurisubharmonic functions $\varphi$ defined on $\Omega$ such that for every open set $G \Subset \Omega$ there exists a plurisubharmonic function $\psi \in \mathcal{F}(\Omega)$ satisfying $\psi \geq \varphi$ on $\Omega$ and $\psi=\varphi$ in $G$.

Definition 3. Let $\mathcal{K} \in\{\mathcal{F}, \mathcal{E}, \mathcal{D}\}$. We denote by $\mathcal{K}^{a}(\Omega)$ the subclass of $\mathcal{K}(\Omega)$ such that the complex Monge-Ampère measure $\left(d d^{c} \text {. }\right)^{n}$ vanishes on all pluripolar sets of $\Omega$.

Let $f \in \mathcal{E}(\Omega)$ and $\mathcal{K} \in\left\{\mathcal{F}^{a}, \mathcal{E}^{a}, \mathcal{D}^{a}, \mathcal{F}, \mathcal{E}, \mathcal{D}\right\}$. Then we say that a plurisubharmonic function $\varphi$ defined on $\Omega$ belongs to $\mathcal{K}(\Omega, f)$ if there exists a function $\psi \in \mathcal{K}(\Omega)$ such that

$$
\psi+f \leq \varphi \leq f \text { in } \Omega
$$

Proposition 1. If $\Omega$ is a bounded hyperconvex domain in $\mathbb{C}^{n}$ then $\mathcal{E}(\Omega)=P S H^{-}(\Omega) \cap \mathcal{D}(\Omega)$.

Proof. See Theorem 2.4 in [6].

Remark 1. (i) If $\Omega$ is an open subset of $\mathbb{C}^{n}$ and $u \in \mathcal{D}(\Omega)$ then $\left.u\right|_{\mathbb{B}}-\sup _{\mathbb{B}} u \in \mathcal{E}(\mathbb{B})$ for all open balls $\mathbb{B} \Subset \Omega$.

(ii) If $\Omega$ is a hyperconvex domain in $\mathbb{C}^{n}$ and $u, f \in \mathcal{E}(\Omega)$ with $u \leq f$ and $h \in \mathcal{F}^{a}(\Omega)$ then $\max (u, f+h) \in \mathcal{F}^{a}(\Omega, f)$.

Next, we recall the following.

Definition 4. Let $\Omega \subset \mathbb{C}^{n}$ and $v \in P S H(\Omega)$. $v$ is said to be a maximal plurisubharmonic function if for all compact subset $K \Subset \Omega$ and for every plurisubharmonic function $w \in$ $P S H(\Omega), w \leq v$ on $\Omega \backslash K$ then $w \leq v$ on $\Omega$.

The family of maximal plurisubharmonic functions on $\Omega$ is denoted by $M P S H(\Omega)$. 
For results concerning to maximal plurisubharmonic functions we refer the readers to [19]. In the case $\Omega \subset \mathbb{C}^{n}$ is a bounded hyperconvex domain and $u \in \mathcal{E}(\Omega)$. Then in [7], Błocki proved that $u \in M P S H(\Omega)$ if and only if it satisfies the homogeneous Monge-Ampère equation $\left(d d^{c} u\right)^{n}=0$.

Proposition 2. Let $\Omega \subset \hat{\Omega}$ be bounded hyperconvex domains in $\mathbb{C}^{n}$. Assume that $u \in \mathcal{F}(\Omega)$ and define

$$
\hat{u}:=\sup \left\{\varphi \in P S H^{-}(\hat{\Omega}): \varphi \leq u \text { on } \Omega\right\} .
$$

If $\left(d d^{c} u\right)^{n}$ is carried by a pluripolar set of $\Omega$ then

$$
\left(d d^{c} \hat{u}\right)^{n}=1_{\Omega}\left(d d^{c} u\right)^{n} \text { in } \hat{\Omega} .
$$

Proof. By Lemma 4.5 in [17] we have $\hat{u} \in \mathcal{F}(\hat{\Omega})$ and $\left(d d^{c} \hat{u}\right)^{n} \leq 1_{\Omega}\left(d d^{c} u\right)^{n}$ in $\hat{\Omega}$. Since $\left(d d^{c} u\right)^{n}$ is carried by a pluripolar set of $\Omega$, Theorem 5.11 in [9] implies that $\left(d d^{c} u\right)^{n}=1_{\{u=-\infty\}}\left(d d^{c} u\right)^{n}$ in $\Omega$. Moreover, since $\hat{u} \leq u$ on $\Omega$, by Lemma 4.1 in [1] we get

$$
1_{\Omega}\left(d d^{c} u\right)^{n}=1_{\Omega \cap\{u=-\infty\}}\left(d d^{c} u\right)^{n} \leq 1_{\Omega \cap\{\hat{u}=-\infty\}}\left(d d^{c} \hat{u}\right)^{n} \leq\left(d d^{c} \hat{u}\right)^{n} \leq 1_{\Omega}\left(d d^{c} u\right)^{n} .
$$

This implies that $\left(d d^{c} \hat{u}\right)^{n}=1_{\Omega}\left(d d^{c} u\right)^{n}$ in $\hat{\Omega}$, and the desired conclusion follows.

3. Weak solutions to the complex Monge-Ampère equations In this section we prove Theorem 1. We need the following auxiliary results.

Lemma 1. Let $\Omega$ be an open set in $\mathbb{C}^{n}$ and let $\mathbb{B} \Subset \Omega$ be an open ball. Assume that $u \in \mathcal{D}(\Omega)$ and $v \in \mathcal{F}(\mathbb{B})$ such that

(i) $\sup _{\mathbb{B}} u<0$;

(ii) $\left(d d^{c} v\right)^{n}$ is carried by a pluripolar set $E \subset \mathbb{B}$;

(iii) $\left(d d^{c} u\right)^{n}$ is carried by a pluripolar set $F \subset \Omega \backslash E$;

(iv) $w:=\sup \{\varphi \in P S H(\Omega): \varphi \leq u$ on $\Omega$ and $\varphi \leq v$ on $\mathbb{B}\} \in \mathcal{D}(\Omega)$.

Then, $\left(d d^{c} w\right)^{n}=\left(d d^{c} u\right)^{n}+1_{\mathbb{B}}\left(d d^{c} v\right)^{n}$ in $\Omega$.

Proof. Without loss of generality we can assume that $E \subset \mathbb{B} \cap\{v=-\infty\}$ and $F \subset(\Omega \backslash E) \cap$ $\{u=-\infty\}$. Let $\left\{\Omega_{j}\right\}$ be an increasing sequence of open sets in $\mathbb{C}^{n}$ such that $\mathbb{B}=\mathbb{B}(a, r) \Subset$ $\Omega_{j} \Subset \Omega_{j+1} \Subset \Omega$ and $\Omega=\bigcup_{j=1}^{\infty} \Omega_{j}$. By the local property of the class $\mathcal{D}(\Omega)$ we may assume that $w \in \mathcal{D}\left(\Omega_{j}\right)$. Set $u_{j}=\left.u\right|_{\Omega_{j}}$. Define

$$
w_{j}:=\sup \left\{\varphi \in P S H\left(\Omega_{j}\right): \varphi \leq u_{j} \text { on } \Omega_{j} \text { and } \varphi \leq v \text { on } \mathbb{B}\right\} .
$$

From $w \leq w_{j}$ on $\Omega_{j}$ then $w_{j} \in \mathcal{D}\left(\Omega_{j}\right)$ and $w_{j} \searrow w$ in $\Omega$. Hence, Proposition 2.1 in [5] implies that $\left(d d^{c} w_{j}\right)^{n}$ converges to $\left(d d^{c} w\right)^{n}$ in the weak ${ }^{*}$-topology. Replacing $\Omega$ by $\Omega_{j}$ if necessary, we can assume that $\Omega$ is bounded and $u$ is a plurisubharmonic function on an open neighborhood of $\bar{\Omega}$. We first prove that

$$
\left(d d^{c} w\right)^{n}=0 \text { on }(\{w<u\} \cap \Omega \backslash \overline{\mathbb{B}}) \cup(\{w<\min (u, v)\} \cap \mathbb{B}) .
$$

Indeed, let $\left\{v_{j}\right\} \subset \mathcal{E}_{0}(\mathbb{B}) \cap \mathcal{C}(\overline{\mathbb{B}})$ be such that $v_{j} \searrow v$ in $\mathbb{B}$ and let $\left\{u_{j}\right\} \subset P S H(\Omega) \cap \mathcal{C}(\Omega)$ be such that $u_{j} \searrow u$ in $\Omega$ and $\sup _{\mathbb{B}} u_{1}<0$. We set

$$
w_{j}:=\sup \left\{\varphi \in P S H(\Omega): \varphi \leq h_{j} \text { in } \Omega\right\},
$$


where

$$
h_{j}= \begin{cases}\min \left(u_{j}, v_{j}\right) & \text { on } \mathbb{B}, \\ u_{j} & \text { on } \Omega \backslash \mathbb{B} .\end{cases}
$$

Since $u_{j} \leq u_{1}<0=v_{j}$ on $\partial \mathbb{B}$ so $h_{j} \in \mathcal{C}(\Omega)$. Then, $w_{j} \in P S H(\Omega)$ and $w_{j} \searrow w$ in $\Omega$. Thanks to Corollary 9.2 in [4] we obtain that $\left(d d^{c} w_{j}\right)^{n}=0$ on $\left\{w_{j}<h_{j}\right\}$. Let $j \rightarrow \infty$, by [11] we get the equality (2).

Next, we claim that

$$
\left(d d^{c} w\right)^{n}=0 \text { in }\{u>-\infty\} \cap\{u=w\}
$$

Indeed, let $K \subset\{u>-\infty\} \cap\{u=w\}$ be a compact set. Since $K \subset\left\{w+\frac{1}{j}>u\right\}$, by Theorem 4.1 in [18] and using the hypotheses we have

$$
\int_{K}\left(d d^{c} w\right)^{n}=\lim _{j \rightarrow+\infty} \int_{K}\left(d d^{c} \max \left(w+\frac{1}{j}, u\right)\right)^{n} \leq \int_{K}\left(d d^{c} \max (w, u)\right)^{n}=\int_{K}\left(d d^{c} u\right)^{n} .
$$

Moreover, since $\left(d d^{c} u\right)^{n}$ is carried by a pluripolar set $F$, we infer

$$
\int_{K}\left(d d^{c} w\right)^{n} \leq \int_{F \cap\{u>-\infty\}}\left(d d^{c} u\right)^{n}=0 .
$$

Hence, $\int_{K}\left(d d^{c} w\right)^{n}=0$. It follows that $\left(d d^{c} w\right)^{n}=0$ in $\{u>-\infty\} \cap\{u=w\}$, and the desired conclusion follows. Similarly, we also have

$$
\left(d d^{c} w\right)^{n}=0 \text { in } \mathbb{B} \cap\{v>-\infty\} \cap\{v=w\} .
$$

Combining this with (2) and (3) we infer that

$$
\left(d d^{c} w\right)^{n}=0 \text { on }(\{u>-\infty\} \cap \Omega \backslash \overline{\mathbb{B}}) \cup(\{\min (u, v)>-\infty\} \cap \mathbb{B}) .
$$

Now, let $R>0$ be such that $\Omega \Subset \mathbb{B}(0, R)$ and define

$$
\hat{v}:=\sup \left\{\varphi \in P S H^{-}(\mathbb{B}(0, R)): \varphi \leq v \text { on } \mathbb{B}\right\} .
$$

Proposition 2 implies that $\hat{v} \in P S H^{-}(\Omega) \cap \mathcal{D}(\Omega)$ and

$$
\left(d d^{c} \hat{v}\right)^{n}=1_{\mathbb{B}}\left(d d^{c} v\right)^{n}=1_{E}\left(d d^{c} v\right)^{n} \text { in } \Omega .
$$

We claim that

$$
\left(d d^{c} w\right)^{n}=\left(d d^{c}(u+\hat{v})\right)^{n}=\left(d d^{c} u\right)^{n} \text { on }\{u=-\infty\} \backslash E .
$$

Indeed, let $K \subset\{u=-\infty\} \backslash E$ be a compact set. Since $\left(d d^{c} \hat{v}\right)^{n}=1_{E}\left(d d^{c} v\right)^{n}$, and $K \subset\{u=$ $-\infty\} \backslash E$ then

$$
\int_{K}\left(d d^{c} \hat{v}\right)^{n}=0
$$

Lemma 4.1 and Lemma 4.4 in [1] imply that

$$
\int_{K}\left(d d^{c} u\right)^{n} \leq \int_{K}\left(d d^{c}(u+\hat{v})\right)^{n}=\sum_{j=0}^{n}\left(\begin{array}{c}
n \\
j
\end{array}\right) \int_{K}\left(d d^{c} u\right)^{j} \wedge\left(d d^{c} \hat{v}\right)^{n-j} \leq
$$




$$
\leq \sum_{j=0}^{n}\left(\begin{array}{l}
n \\
j
\end{array}\right)\left(\int_{K}\left(d d^{c} u\right)^{n}\right)^{\frac{j}{n}}\left(\int_{K}\left(d d^{c} \hat{v}\right)^{n}\right)^{\frac{n-j}{n}}=\int_{K}\left(d d^{c} u\right)^{n} .
$$

It follows that

$$
\int_{K}\left(d d^{c}(u+\hat{v})\right)^{n}=\int_{K}\left(d d^{c} u\right)^{n} .
$$

Moreover, since $u+\hat{v} \leq w \leq u$ in $\Omega$, then Theorem 4.1 in [1] implies that

$$
\int_{K}\left(d d^{c} u\right)^{n} \leq \int_{K}\left(d d^{c} w\right)^{n} \leq \int_{K}\left(d d^{c}(u+\hat{v})\right)^{n}=\int_{K}\left(d d^{c} u\right)^{n},
$$

and the required conclusion follows. Similarly, we also have

$$
\left(d d^{c} w\right)^{n}=\left(d d^{c}(u+\hat{v})\right)^{n}=\left(d d^{c} \hat{v}\right)^{n}=\left(d d^{c} v\right)^{n} \text { on }(\mathbb{B} \cap\{v=-\infty\}) \backslash F .
$$

Combining this with (4), (5) and using the hypotheses we infer

$$
\left(d d^{c} w\right)^{n}=\left(d d^{c} u\right)^{n}+1_{\mathbb{B}}\left(d d^{c} v\right)^{n} \text { in } \Omega \backslash \partial \mathbb{B} .
$$

Let $\mathbb{B}^{\prime}$ be an open ball such that $\mathbb{B} \Subset \mathbb{B}^{\prime} \Subset \Omega$ and $\sup _{\mathbb{B}^{\prime}} u<0$. Put

$$
v^{\prime}:=\sup \left\{\varphi \in P S H^{-}\left(\mathbb{B}^{\prime}\right): \varphi \leq v \text { on } \mathbb{B}\right\} .
$$

By Proposition 2 we have $v^{\prime} \in \mathcal{F}\left(\mathbb{B}^{\prime}\right)$ and $\left(d d^{c} v^{\prime}\right)^{n}=1_{\mathbb{B}}\left(d d^{c} v\right)^{n}$ in $\mathbb{B}^{\prime}$. Since $w \leq u<0$ in $\mathbb{B}^{\prime}$ and $w \leq v$ in $\mathbb{B}$ so $w \leq v^{\prime}$ on $\mathbb{B}^{\prime}$. This implies that

$$
w=\sup \left\{\varphi \in P S H(\Omega): \varphi \leq u \text { on } \Omega \text { and } \varphi \leq v^{\prime} \text { on } \mathbb{B}^{\prime}\right\} .
$$

Applying (6) we get that

$$
\left(d d^{c} w\right)^{n}=\left(d d^{c} u\right)^{n}+1_{\mathbb{B}^{\prime}}\left(d d^{c} v^{\prime}\right)^{n} \text { in } \Omega \backslash \partial \mathbb{B}^{\prime} .
$$

Therefore, $\left(d d^{c} w\right)^{n}=\left(d d^{c} u\right)^{n}+1_{\mathbb{B}}\left(d d^{c} v\right)^{n}$ in $\Omega$.

Lemma 2. Let $\Omega$ be an open set in $\mathbb{C}^{n}$ and let $u, v \in \mathcal{D}(\Omega)$ be such that $u \leq v$ in $\Omega$. Assume that $\left(d d^{c} v\right)^{n}$ is carried by a pluripolar set of $\Omega$ and $\mu$ is a non-negative Radon measure defined in $\Omega$ with $\mu \leq 1_{\{u>-\infty\}}\left(d d^{c} u\right)^{n}$. Then, for every open ball $\mathbb{B} \Subset \Omega$, there exists $w \in \mathcal{D}(\Omega)$ satisfying

(i) $u \leq w \leq v$ on $\Omega$;

(ii) $\left(d d^{c} w\right)^{n} \geq \mu$ in $\Omega$;

(iii) $\left(d d^{c} w\right)^{n}=\mu+\left(d d^{c} v\right)^{n}$ in $\mathbb{B}$.

Proof. Fix a ball $\mathbb{B} \Subset \Omega$. Let $\mathbb{B}^{\prime}$ be an open ball such that $\mathbb{B} \Subset \mathbb{B}^{\prime} \Subset \Omega$ and $u \in \mathcal{E}\left(\mathbb{B}^{\prime}\right)$. Without loss of generality we may assume that $\left(d d^{c} v\right)^{n}$ is carried by $\{v=-\infty\}$. Define

$$
\begin{gathered}
g:=\left(\sup \left\{\varphi \in P S H^{-}\left(\mathbb{B}^{\prime}\right): \varphi \leq u \text { on } \mathbb{B}^{\prime} \backslash \mathbb{B}\right\}\right)^{*}, \\
f:=\sup \left\{\varphi \in P S H^{-}\left(\mathbb{B}^{\prime}\right): \varphi \leq \min (g, v) \text { on } \mathbb{B}^{\prime}\right\} .
\end{gathered}
$$


Then, $g \in \mathcal{E}\left(\mathbb{B}^{\prime}\right) \cap M P S H(\mathbb{B})$. Since $u \leq v$ in $\Omega$ so $u \leq f \leq v$ in $\mathbb{B}^{\prime}$ and $f=g=u$ on $\mathbb{B}^{\prime} \backslash(\mathbb{B} \cup E)$ for some pluripolar set $E \subset \partial \mathbb{B}$. By Lemma 4.1 in [1], Corollary 3.1 in [15] and using the hypotheses we have

$$
\left(d d^{c} v\right)^{n}=1_{\{v=-\infty\}}\left(d d^{c} v\right)^{n} \leq 1_{\{f=-\infty\}}\left(d d^{c} f\right)^{n} \leq\left(d d^{c} f\right)^{n} \leq\left(d d^{c} v\right)^{n}+\left(d d^{c} g\right)^{n}=\left(d d^{c} v\right)^{n}
$$

on $\mathbb{B}$. It follows that $\left(d d^{c} f\right)^{n}=\left(d d^{c} v\right)^{n}$ on $\mathbb{B}$.

Now, since the measure $1_{\{u>-\infty\}}\left(d d^{c} u\right)^{n}$ vanishes on all pluripolar sets of $\Omega$ and $\mu \leq$ $1_{\{u>-\infty\}}\left(d d^{c} u\right)^{n}$ in $\Omega$ so by Lemma 5.14 in [9] there exists $h \in \mathcal{F}^{a}(\mathbb{B})$ such that $\left(d d^{c} h\right)^{n}=$ $\mu$ in $\mathbb{B}$. It is easy to see that $\max (u, h+f) \in \mathcal{F}^{a}(\mathbb{B}, f)$ and

$$
\left(d d^{c} f\right)^{n} \leq \mu+\left(d d^{c} v\right)^{n} \leq\left(d d^{c} \max (u, h+f)\right)^{n} \text { in } \mathbb{B} .
$$

Thanks to Lemma 4.1 in [16] there is $\psi \in \mathcal{F}^{a}(\mathbb{B}, f)$ such that $u \leq \psi \leq f$ and

$$
\left(d d^{c} \psi\right)^{n}=\mu+\left(d d^{c} v\right)^{n} \text { in } \mathbb{B} .
$$

Let $w$ be the smallest plurisubharmonic majorant of the function

$$
\eta= \begin{cases}\psi & \text { in } \mathbb{B}, \\ u & \text { in } \Omega \backslash \mathbb{B} .\end{cases}
$$

Since $u \leq \psi \leq f \leq g$ on $\mathbb{B}$, we have $w \in \mathcal{D}(\Omega)$ and $u \leq w \leq v$ in $\Omega$. It is easy to see that

$$
\left(d d^{c} w\right)^{n} \geq \mu \text { in } \Omega \backslash \mathbb{B}
$$

and

$$
\left(d d^{c} w\right)^{n}=\mu+\left(d d^{c} v\right)^{n} \text { on } \mathbb{B} .
$$

Indeed, by the definition of $w$ we note that $w=u$ in the interior of $\Omega \backslash \mathbb{B}$ and $w=\psi$ in $\mathbb{B}$. Hence, we have $\left(d d^{c} w\right)^{n} \geq \mu$ on the interior of $\Omega \backslash \mathbb{B}$ and (8) holds. In order to prove $\left(d d^{c} w\right)^{n} \geq \mu$ on $\Omega \backslash \mathbb{B}$ it suffices to prove $\left(d d^{c} w\right)^{n} \geq \mu$ on $\partial \mathbb{B}$. By the definition of $w$ it follows that $w=u$ on $\partial \mathbb{B} \backslash E$, where $E$ is a pluripolar subset of $\partial \mathbb{B}$ containing $\{u=-\infty\}$. Let $K \subset \partial \mathbb{B} \backslash E$ be a compact set. Since $K \subset\left\{u+\frac{1}{j}>w\right\}$, by Theorem 4.1 in [18] we have

$$
\mu(K) \leq \int_{K}\left(d d^{c} u\right)^{n}=\lim _{j \rightarrow+\infty} \int_{K}\left(d d^{c} \max \left(u+\frac{1}{j}, w\right)\right)^{n} \leq \int_{K}\left(d d^{c} \max (u, w)\right)^{n}=\int_{K}\left(d d^{c} w\right)^{n} .
$$

It follows that $\left(d d^{c} w\right)^{n} \geq \mu$ on $\partial \mathbb{B} \backslash E$. Hence, $\left(d d^{c} w\right)^{n} \geq \mu$ on $\partial \mathbb{B}$. Combining this with (7) and (8) we obtain $\left(d d^{c} w\right)^{n} \geq \mu$ in $\Omega$. The proof is complete.

Proof of Theorem 1. Let $\left\{\mathbb{B}_{j}\right\}$ be a sequence of open balls such that $\mathbb{B}_{j} \Subset \Omega$ and $\Omega=$ $\bigcup_{j=1}^{\infty} \mathbb{B}_{j}$. Put $\mathbb{B}_{0}:=\varnothing$. We first claim that there exists a decreasing sequence $\left\{f_{j}\right\} \subset \mathcal{D}(\Omega)$ such that $u \leq f_{j} \leq v$ and

$$
\left(d d^{c} f_{j}\right)^{n}=1_{\left(\bigcup_{k=0}^{j-1} \mathbb{B}_{k}\right) \cap\{u=-\infty\}} \mu \text { on } \Omega .
$$

Indeed, let $f_{1}:=v$. Then, $f_{1} \in \mathcal{D}(\Omega)$ and $\left(d d^{c} f_{1}\right)^{n}=0$ in $\Omega$. Let $j \geq 1$ be an integer number. Assume by induction that we have determined $f_{j}$. We find $f_{j+1}$ as follows. Let $\mathbb{B}_{j}^{\prime}$ be an 
open ball such that $\mathbb{B}_{j} \Subset \mathbb{B}_{j}^{\prime} \Subset \Omega$. By Theorem 4.14 in [1], there exists $v_{j} \in \mathcal{F}\left(\mathbb{B}_{j}^{\prime}\right)$ such that $v_{j} \geq u$ and

$$
\left(d d^{c} v_{j}\right)^{n}=1_{\left(\mathbb{B}_{j} \backslash \bigcup_{k=0}^{j-1} \mathbb{B}_{k}\right) \cap\{u=-\infty\}} \mu \text { on } \mathbb{B}_{j}^{\prime} .
$$

Set $c_{j}:=\sup _{\mathbb{B}_{j}^{\prime}} v+\lambda_{j}$ where $\lambda_{j}$ is chosen such that $c_{j} \geq 0$. Next, we define

$$
f_{j+1}=\sup \left\{\varphi \in P S H(\Omega): \varphi \leq f_{j}-c_{j} \text { in } \Omega \text { and } \varphi \leq v_{j} \text { in } \mathbb{B}_{j}^{\prime}\right\}+c_{j} .
$$

Then we have $u \leq f_{j+1} \leq f_{j} \leq v$ on $\Omega$ and by Theorem 1.2 in [6] we have $f_{j+1} \in \mathcal{D}(\Omega)$. Lemma 1 implies that

$$
\left(d d^{c} f_{j+1}\right)^{n}=\left(d d^{c} f_{j}\right)^{n}+1_{\mathbb{B}_{j}^{\prime}}\left(d d^{c} v_{j}\right)^{n}=1_{\left(\bigcup_{k=0}^{j} \mathbb{B}_{k}\right) \cap\{u=-\infty\}} \mu \text { in } \Omega .
$$

This proves the claim. Put $f:=\lim _{j \rightarrow \infty} f_{j}$ in $\Omega$. Then, $f \in P S H(\Omega)$ and $u \leq f \leq v$ in $\Omega$. By Theorem 1.2 in [6] and using the main result in [11] we infer that $f \in \mathcal{D}(\Omega)$ and

$$
\left(d d^{c} f\right)^{n}=1_{\{u=-\infty\}} \mu \text { in } \Omega .
$$

We now set

$$
w:=\sup \left\{\varphi \in \mathcal{D}(\Omega): \varphi \leq f \text { and }\left(d d^{c} \varphi\right)^{n} \geq 1_{\{u>-\infty\}} \mu\right\} .
$$

Because $u \leq w \leq f \leq v$ in $\Omega$, by Theorem 1.2 in [6] we have $w \in \mathcal{D}(\Omega)$. Since the measure $1_{\{u>-\infty\}} \mu$ vanishes on pluripolar sets of $\Omega$, by Proposition 4.3 in [18] and using the Choquet lemma we can choose a increasing sequence $\left\{\varphi_{j}\right\} \subset \mathcal{D}(\Omega)$ such that $\varphi_{j} \nearrow w$ a.e. in $\Omega$ and

$$
\left(d d^{c} \varphi_{j}\right)^{n} \geq 1_{\{u>-\infty\}} \mu \text { in } \Omega \text {. }
$$

By the main result in [11] we note that $\left(d d^{c} \varphi_{j}\right)^{n} \rightarrow\left(d d^{c} w\right)^{n}$ weakly in $\Omega$, and hence,

$$
\left(d d^{c} w\right)^{n} \geq 1_{\{u>-\infty\}} \mu \text { in } \Omega .
$$

Let $\mathbb{B} \Subset \Omega$ be an open ball. By Lemma 2 there exists $\psi \in \mathcal{D}(\Omega)$ such that

(i) $w \leq \psi \leq f$ on $\Omega$;

(ii) $\left(d d^{c} \psi\right)^{n} \geq 1_{\{u>-\infty\}} \mu$ in $\Omega$;

(iii) $\left(d d^{c} \psi\right)^{n}=1_{\{u>-\infty\}} \mu+\left(d d^{c} f\right)^{n}$ in $\mathbb{B}$.

From the definition of $w$ it is easy to see that $w=\psi$ in $\Omega$, and therefore, by (iii) we have

$$
\left(d d^{c} w\right)^{n}=1_{\{u>-\infty\}} \mu+\left(d d^{c} f\right)^{n}=\mu \text { in } \mathbb{B},
$$

and the desired conclusion follows.

Acknowledgements. This work was done during the stay of the first-named author at Vietnam Institute for Advanced Study in Mathematics (VIASM). He would like to express his gratitude to the Institute for the support. The authors thank Dr. Nguyen Xuan Hong for useful discussion in preparation of the paper. This research is funded by the Vietnam Ministry of Education and Training. 


\section{REFERENCES}

1. P. Åhag, U. Cegrell, R. Czyż, P.H. Hiep, Monge-Ampère measures on pluripolar sets, J. Math. Pures Appl., 92 (2009), 613-627.

2. P. Åhag, R. Czyż, Kolodziej's subsolution theorem for unbounded pseudoconvex domains, Universitatis Iagellonicae Acta Mathematica, 50 (2012), 7-23.

3. E. Bedford, B.A. Taylor, The Dirichlet problem for a complex Monge-Ampère operator, Invent. Math., 37 (1976), 1-44.

4. E. Bedford, B.A. Taylor, A new capacity for plurisubharmonic functions, Acta Math., 149 (1982), 1-41.

5. Z. Błocki, On the definition of the Monge-Ampère operator in $\mathbb{C}^{2}$, Math. Ann., 328 (2004), 415-423.

6. Z. Błocki, The domain of definition of the complex Monge-Ampère operator, Amer. J. Math., 128 (2006), 519-530.

7. Z. Błocki, A note on maximal plurisubharmonic functions, Uzbek. Math. J., 1 (2009), 28-32.

8. U. Cegrell, Pluricomplex energy, Acta Math., 180 (1998), 187-217.

9. U. Cegrell, The general definition of the complex Monge-Ampère operator, Ann. Inst. Fourier., 54 (2004), 159-179.

10. U. Cegrell, A general Dirichlet problem for the complex Monge-Ampère operator, Ann. Polon. Math., 94 (2008), 131-147.

11. U. Cegrell, Convergence in capacity, Canad. Math. Bull., 55 (2012), 242-248.

12. L.M. Hai, N.V. Khue, P.H. Hiep, The complex Monge-Ampère operator on bounded domains in $\mathbb{C}^{n}$, Result. Math., 54 (2009), 309-328.

13. L.M. Hai, N.V. Trao, N.X. Hong, The complex Monge-Ampère equation in unbounded hyperconvex domains in $\mathbb{C}^{n}$, Complex Variables and Elliptic Equations, 59 (2014), №12, 1758-1774.

14. L.M. Hai, T.V. Thuy, N.X. Hong, A note on maximal subextensions of plurisubharmonic functions, Acta Math Vietnam, 43 (2018), 137-146.

15. N.X. Hong, Monge-Ampère measures of maximal subextensions of plurisubharmonic functions with given boundary values, Complex Var. Elliptic Equ., 60 (2015), №3, 429-435.

16. N.X. Hong, N.V. Trao, T.V. Thuy, Convergence in capacity of plurisubharmonic function functions with given boundary values, Inter. J. Math, 28, №3, (2017) 14 p.

17. P.H. Hiep, Pluripolar sets and the subextension in Cegrell's classes, Complex Var. Elliptic Equ., 53 (2008), №7, 675-684.

18. N.V. Khue, P.H. Hiep, A comparison principle for the complex Monge-Ampère operator in Cegrell's classes and applications, Trans. Am. Math. Soc., 361 (2009), 5539-5554.

19. M. Klimek, Pluripotential Theory, The Clarendon Press Oxford University Press, New York, 1991, Oxford Science Publications.

20. S. Kołodziej, The range of the complex Monge-Ampère operator, II, Indiana U. Math. J., 44 (1995), 765-782.

21. Y.T. Siu, Extension of meromorphic maps into Kähler manifolds, Ann. of Math., 102 (1975), №2, 421462.

Hanoi Architectural University, Hanoi, Viet Nam vuquanhau.edu@gmail.com

Department of Mathematics, Hanoi National University of Education Xuan Thuy Street, Cau Giay, Hanoi, Viet Nam mauhai@fpt.vn 